\title{
SYMMETRIES OF LINKS
}

\author{
BY \\ W. C. WHITTEN, JR.
}

In this paper certain properties called symmetries are defined for links, and the problem of determining those links admitting a particular symmetry is attacked. The problems of symmetry are the generalization to links of the problems of amphichaerality and invertibility of knots which, because of Trotter's proof that there are noninvertible knots [13], are now fairly well under control. A link of two components is called interchangeable if it possesses a special type of symmetry, and certain invariants of interchangeability of a link are given and examined.

By a link of $\mu$ components we shall understand the union of $\mu$ oriented, ordered, and disjoint knots $K_{1}, \ldots, K_{\mu}$ tamely imbedded in the oriented 3-sphere $S$. Two links, $L$ and $L^{\prime}$, are of the same (oriented) type if and only if there is an orientation-preserving autohomeomorphism $\phi$ of $S$ which takes $L$ onto $L^{\prime}$ such that $\phi\left(+K_{\alpha}\right)$ $=+K_{\alpha}^{\prime}$ for each $\alpha$; that is, the orientation of $\phi\left(+K_{\alpha}\right)$ is to match that of $K_{\alpha}^{\prime}$ for each $\alpha$.

The problems of amphichaerality and invertibility of knots (see either [1] or [13]) have been generalized to links by R. H. Fox as follows. Let $L$ be a link of $\mu$ components, $S_{\mu}$ the symmetric group of degree $\mu$, and $Z_{2}^{\mu+1}$ the direct product of $\mu+1$ copies of the multiplicative group $Z_{2}=\{-1,1\}$. Define $\Gamma_{\mu}$ as a split extension $1 \rightarrow Z_{2}^{\mu+1} \rightarrow \Gamma_{\mu} \rightarrow S_{\mu} \rightarrow 1$ with isomorphism $W$ taking $S_{\mu}$ into Aut $\left(Z_{2}^{\mu+1}\right)$ by $W(p)=W_{p}$ for each $p$ in $S_{\mu}$, where $W_{p}$ is given by

$$
W_{p}\left(\varepsilon_{0}, \varepsilon_{1}, \ldots, \varepsilon_{\mu}\right)=\left(\varepsilon_{0}, \varepsilon_{p(1)}, \ldots, \varepsilon_{p(\mu)}\right)
$$

for each $\left(\varepsilon_{0}, \varepsilon_{1}, \ldots, \varepsilon_{\mu}\right)$ in $Z_{2}^{\mu+1}$; that is, $S_{\mu}$ permutes the last $\mu$ factors of $Z_{2}^{\mu+1}$. $\gamma=\left(\varepsilon_{0}, \varepsilon_{1}, \ldots, \varepsilon_{\mu}, p\right)$, where $\varepsilon_{i}= \pm 1$ and $p$ is a permutation on $\{1,2, \ldots, \mu\}$, is an element of $\Gamma_{\mu}$. We shall say that $L$ admits $\gamma$ if $L^{\gamma}=\varepsilon_{1} K_{p(1)} \cup \cdots \cup \varepsilon_{\mu} K_{p(\mu)}$ is of the same type as $L=+K_{1} \cup \cdots \cup+K_{u}$; that is, if there is an autohomeomorphism $\psi$ of $S$ such that $\psi(+S)=\varepsilon_{0} S$, and $\psi\left(+K_{\alpha}\right)=\varepsilon_{\alpha} K_{p(\alpha)}$ for each $\alpha$.

The elements $\gamma$ admitted by $L$ form a subgroup $\Sigma(L)$ of $\Gamma_{\mu}$, the group of symmetries of $L . \Sigma(L)$ is unaltered by change of orientation of $S$ or of any $K_{\alpha}$, but change in the orientation of any $K_{\alpha}$ or in the order of $K_{1}, \ldots, K_{\mu}$ induces an inner automorphism of $\Gamma_{\mu}$ which replaces $\Sigma(L)$ by a conjugate subgroup of $\Gamma_{\mu}$.

Three questions which now arise are:

(1) What links admit a given $\gamma$ ?

(2) What links have a given subgroup $\Sigma$ of $\Gamma_{\mu}$ as their group of symmetries?

Presented to the Society, April 22, 1966 under the title On the interchangeability of 2-links. II; received by the editors August 10, 1967. 
(3) In particular, for what subgroups $\Sigma$ of $\Gamma_{\mu}$ does there exist a link $L$ for which $\Sigma(L)=\Sigma$ ?

For $\mu=1$, the five (proper and improper) subgroups of $\Gamma_{1}=Z_{2} \times Z_{2}$ describe five possible properties of a knot: 1 . nonamphichaeral and noninvertible; 2 . nonamphichaeral and invertible; 3. +amphichaeral and noninvertible; 4. -amphichaeral and noninvertible; 5. amphichaeral and invertible. For $\mu=2$, a link is called interchangeable (see problem 11 of [2]) if and only if it admits any $\gamma$ of the form $\left(\varepsilon_{0}, \varepsilon_{1}, \varepsilon_{2},(12)\right)$.

In $\S 1$ of this paper, the problem (1) above is reduced to the (oriented) type problem and the special case of problem (1) in which the link $L$ is unsplittable and prime. As a corollary to Theorem 2 of $\S 1$, necessary and sufficient conditions are obtained for an unsplittable link of two components to be interchangeable. These conditions can be used on occasion to show that certain links are not interchangeable.

In $\S 2$, the cyclic covering spaces of $S$ branched over a link are brought into play. If $M_{g}$ denotes the $g$-fold cyclic covering space of $S$ branched over $L=K_{1}$ $\cup \cdots \cup K_{\mu}$ and $\tilde{L}=\tilde{K}_{1} \cup \ldots \cup \tilde{K}_{\mu} \subset M_{g}$ lies over $L$ with $\tilde{K}_{i}$ lying over $K_{i},(i=1, \ldots$, $\mu)$, then $H_{1}\left(\left(M_{g}-\tilde{L}\right) \cup \widetilde{K}_{i}\right)$ and $H_{1}\left(\left(M_{g}-\tilde{L}\right) \cup \widetilde{K}_{j}\right)$ are shown to be isomorphic. As an immediate corollary to the proof of this fact, $H_{1}\left(M_{g}-\tilde{L}\right)$ must be isomorphic to the direct sum of the infinite cyclic group and $H_{1}\left(\left(M_{g}-\tilde{L}\right) \cup \widetilde{K}_{i}\right)$. For interchangeability invariants of $L=K_{1} \cup K_{2}$, we go to the various unbranched cyclic covering spaces of $M_{g}-\tilde{K}_{1}$ and $M_{g}-\tilde{K}_{2}$.

Examples are given in $\$ 3$.

I wish to express my appreciation to Professor R. H. Fox of Princeton University for his generous assistance. Also, I would like to thank Professor J. W. Milnor for a helpful suggestion.

\section{Reduction of problem (1).}

THEOREM 1. If $L$ is a splittable link of $\mu$ components and its unsplittable parts are $L_{i}=K_{v_{i-1}+1} \cup \cdots \cup K_{v_{i}},(i=1, \ldots, m)$, then in order that $L$ admit $\gamma=\left(\varepsilon_{0}, \varepsilon_{1}, \ldots\right.$, $\left.\varepsilon_{\mu}, p\right)$ it is necessary and sufficient that $p$ induce a permutation

$$
\left(\begin{array}{c}
T_{1} \cdots T_{m} \\
T_{q(1)} \cdots T_{q(m)}
\end{array}\right)
$$

of the sets $T_{i}=\left\{\nu_{i-1}+1, \ldots, \nu_{i}\right\}$ such that for each $i, L_{q(i)}$ and $L_{i}^{\gamma_{1}}$ are equal as point sets, and $L_{i}^{\gamma_{i}}$ be of the same type as $L_{i}$, where

and

$$
\gamma_{i}=\left(\varepsilon_{0}, \varepsilon_{v_{i-1}+1}, \ldots, \varepsilon_{v_{i}}, p_{i}\right)
$$

$$
p_{i}=\left(\begin{array}{c}
v_{i-1}+1 \cdots v_{i} \\
p\left(v_{i-1}+1\right) \cdots p\left(v_{i}\right)
\end{array}\right) \text {. }
$$

In particular, for those $i$ for which $q(i)=i$, the requirement is that $L_{i}$ admit the element $\gamma_{i}$ of $\Gamma_{v_{i}-v_{i-1}}$. 
Proof. The proof is quite simple. Since the necessity is obvious, it only remains to prove that the condition is sufficient. All maps are to be autohomeomorphisms of $S$.

Since $L$ is splittable with unsplittable parts $L_{1}, \ldots, L_{m}$, there exist (tame) 3-cells $Q_{1}, \ldots, Q_{m}$ in $S$ with $L_{i} \subset \operatorname{Int}\left(Q_{i}\right)$ and $Q_{i} \cap Q_{j}=\varnothing$ for $i \neq j$. There is a map $\psi_{1}$ such that $\psi_{1}(+S)=\varepsilon_{0} S$ and $\psi_{1}\left(Q_{i}\right)=Q_{q(i)}$ for each $i=1, \ldots, m$. Finally, since $L_{i}^{\gamma_{1}}$ is of the same type as $L_{i}$ for each $i$, there exists a map $\psi_{2}$ which acts as the identity on $S-\left(\right.$ Int $\left.\left(Q_{1}\right) \cup \cdots \cup \operatorname{Int}\left(Q_{m}\right)\right)$ such that for the mapping $\psi=\psi_{2} \psi_{1}, \psi(+S)=\varepsilon_{0} S$ and $\psi\left(+K_{\alpha}\right)=\varepsilon_{\alpha} K_{p(\alpha)}$ for each $\alpha=1, \ldots, \mu$.

Corollary. A splittable link of two components is interchangeable if and only if its components are of the same knot type.

Links $L$ and $L^{\prime}$ will be called equivalent (denoted by $L \approx L^{\prime}$ ), if there is an orientation-preserving autohomeomorphism $\theta$ taking $L$ onto $L^{\prime}$ such that $\theta$ restricted to each component of $L$ is also orientation-preserving. $\theta$ is not required to preserve the order of the components of $L$.

REMARK. All 3-cells and 2-spheres mentioned are to be considered as tamely imbedded in $S$.

Let $L$ be an unsplittable link and let $Q$ be a 3-cell whose boundary 2-sphere Bd $(Q)$ intersects $L$ in exactly two points $a$ and $b$ of some component, say $K_{\alpha}$, of $L$ (that is, $L \cap \mathrm{Bd}(Q)=\{a, b\} \subset K_{\alpha}$ ) in such a way that points of $L$ belong to both the interior and exterior of $Q$. If $q$ is a simple arc (to be called a connection arc) on $\mathrm{Bd}(Q)$ whose endpoints are $a$ and $b$, then for appropriate orientations of $q$, $L_{1}=[L \cap(S-Q)] \cup q$ and $L_{2}=[L \cap \operatorname{Int}(Q)] \cup q$ are links which are uniquely determined by $L$ and $\mathrm{Bd}(Q)$ up to equivalence. We set $k_{1}=\left[K_{\alpha} \cap(S-Q)\right] \cup q$ and $k_{2}=\left[K_{\alpha} \cap \operatorname{Int}(Q)\right] \cup q$, noting that $k_{1}$ and $k_{2}$ are components of the links $L_{1}$ and $L_{2}$, respectively, and that $K_{\alpha}=k_{1} \# k_{2}$ (see p. 139 of [1]).

After Schubert (see p. 142 of [11]), we say that $L$ and $B d(Q)$ form a product representation of $L$ with respect to $K_{\alpha}$, and that $L_{1}$ and $L_{2}$ are factors of $L . L$ is called the product of $L_{1}$ and $L_{2}$ with respect to $k_{1}$ and $k_{2}$, and we write $L=L_{1} \cdot L_{2}$. $L$ is determined up to equivalence by $L_{1}, L_{2}, k_{1}$, and $k_{2}$.

The link $L$ shall be called prime if, whenever $L=L_{1} \cdot L_{2}$, one but not both of $L_{1}$ and $L_{2}$ is a trivial knot. A trivial knot will be interpreted as the empty product of prime links, while a prime link is treated as a product of prime links with one factor. It has been proved [7] that each unsplittable link $L$ is the product of a finite collection of prime links which are uniquely determined by $L$ up to equivalence. Conversely, $L$ is determined up to equivalence by its prime factors and an algorithm for the multiplication of these factors.

THEOREM 2. If an unsplittable link $L$ is the product of prime factors $L_{j}=K_{j, \alpha(j, 1)}$ $\cup \cdots \cup K_{j, \alpha\left(j, l_{j}\right)},(j=1, \ldots, n)$, where the indices $\alpha(j, k)$ are inherited from the 
indexing $\alpha$ of the components of $L=\bigcup K_{\alpha}$, then in order that $L$ admit $\gamma=\left(\varepsilon_{0}\right.$, $\left.\varepsilon_{1}, \ldots, \varepsilon_{\mu}, p\right)$ it is necessary and sufficient that $p$ induce a permutation

$$
\left(\begin{array}{c}
V_{1} \cdots V_{n} \\
V_{r(1)} \cdots V_{r(n)}
\end{array}\right)
$$

of the sets $V_{j}=\left\{(j, \alpha(j, 1)), \ldots,\left(j, \alpha\left(j, l_{j}\right)\right)\right\},(j=1, \ldots, n)$, such that for each $j$,

$$
L_{j}^{\gamma_{j}}=\varepsilon_{\alpha(j, 1)} K_{r(j), p_{j}(\alpha(j, 1))} \cup \cdots \cup \varepsilon_{\alpha\left(j, l_{j}\right)} K_{r(j), p_{j}(\alpha(j, l, j))}
$$

be of the same type as $L_{j}$, where $\gamma_{j}=\left(\varepsilon_{0}, \varepsilon_{\alpha(j, 1)}, \ldots, \varepsilon_{\alpha\left(j, l_{j}\right)}, p_{j}\right)$ and

$$
p_{j}=\left(\begin{array}{c}
\alpha(j, 1) \cdots \alpha\left(j, l_{j}\right) \\
p(\alpha(j, 1)) \cdots p\left(\alpha\left(j, l_{j}\right)\right)
\end{array}\right)
$$

In particular, for those $j$ for which $r(j)=j$, the requirement is that $L_{j}$ admit the element $\gamma_{j}$ of $\Gamma_{l_{j}}$.

Proof. Necessity. There is an autohomeomorphism $\psi$ of $S$ such that $\psi(S)=\varepsilon_{0} S$ and $\psi\left(+K_{\alpha}\right)=\varepsilon_{\alpha} K_{p(\alpha)}$ for each $\alpha=1, \ldots, \mu$. Since $L$ is the product of prime factors $L_{1}, \ldots, L_{n}$, (the prime factors of $L$ may be fixed by considering a decomposition system of $L$ (see p. 286 of [7])), then $L^{\gamma}$ is the product of prime factors $\psi\left(L_{1}\right), \ldots$, $\psi\left(L_{n}\right)$. A second prime factorization $L_{1}^{\prime}, \ldots, L_{n}^{\prime}$ of $L^{\gamma}$ may clearly be obtained from $L_{1}, \ldots, L_{n}$ by properly orienting all components of each $L_{i}$. By the unique factorization theorem for links [7] there is a one-to-one correspondence between $\left\{\psi\left(L_{1}\right), \ldots\right.$, $\left.\psi\left(L_{n}\right)\right\}$ and $\left\{L_{1}^{\prime}, \ldots, L_{n}^{\prime}\right\}$ such that corresponding prime factors are equivalent.

Clearly, $\psi\left(L_{j}\right)$ may be written as

$$
\psi\left(L_{\jmath}\right)=K_{j, p(\alpha(j, 1))}^{*} \cup \cdots \cup K_{j, p(\alpha(j, l)))}^{*},
$$

where the indices $p(\alpha(j, k))$ are inherited from the indexing $p(\alpha)$ of the components of $L^{\gamma}=\bigcup_{\alpha=1}^{\mu} \varepsilon_{\alpha} K_{p(\alpha)}$, and $K_{j, p(\alpha(j, k))}^{*}=\psi\left(K_{j, \alpha(j, k)}\right)$. (Note that no two distinct components of $L_{j}$ inherit $\alpha$-indexing from the same component of $L$.) Now given $\psi\left(L_{j}\right)$, the combined proofs of Lemma 3 and the unique factorization theorem of [7] construct (by use of the well-known methods of Schubert [10]) an orientationpreserving autohomeomorphism $\theta_{j}$ of $S$ taking $\psi\left(L_{j}\right)$ onto one of the links $L_{1}^{\prime}, \ldots, L_{n}^{\prime}$ in such a way that the $\alpha$-indexing of $\theta_{j}\left(K_{j, p(\alpha(j, k))}^{*}\right)$ is precisely $p(\alpha(j, k))$ for each $k=1, \ldots, l_{j}$. If then $\theta_{j} \psi\left(L_{j}\right)=L_{r(j)}^{\prime}$, it follows from the construction of $\theta_{j}$ that $\theta_{j}\left(K_{j, p(\alpha(j, k))}^{*}\right)=\varepsilon_{\alpha(j, k)} K_{r(j), p(\alpha(j, k))},\left(k=1, \ldots, l_{j}\right)$. Again from the construction of the $\theta_{j}$, we have $\theta_{i} \psi\left(L_{i}\right)=\theta_{j} \psi\left(L_{j}\right)$ only if $i=j$, and the necessity of the condition is proved.

In particular, in those cases for which $r(j)=j$, each $p(\alpha(j, k))$ must clearly belong to $\left\{\alpha(j, 1), \ldots, \alpha\left(j, l_{j}\right)\right\}$, so that $L_{j}$ admits $\gamma_{j}$ of $\Gamma_{l,}$.

Sufficiency. The proof proceeds by induction on $n$, the number of prime factors of $L$. For $n=1$ there is nothing to prove.

If $n=2, L$ is the product of prime factors $L_{1}$ and $L_{2}$. There is a 3-cell $Q$ such that $L$ and $\mathrm{Bd}(Q)$ form a product representation of $L$ with respect to some component 
$K_{\alpha}$ of $L$, and such that $L_{1}=[L \cap \operatorname{Int}(Q)] \cup q$ and $L_{2}=[L \cap(S-Q)] \cup q . q$ is a connection $\operatorname{arc}$ on $\mathrm{Bd}(Q)$ appropriately oriented in each case.

Let $\psi_{1}$ be an autohomeomorphism of $S$ such that $\psi_{1}(S)=\varepsilon_{0} S$. By hypothesis there is an orientation-preserving autohomeomorphism $\psi_{2}$ of $S$ taking $\psi_{1}\left(L_{1}\right)$ onto $L_{1}^{\gamma_{1}}$. It may be arranged (see the proof of Theorem $3^{\prime}$ of [10]) that $\psi_{2}$ take $\psi_{1}(\mathrm{Bd}(Q))$ onto $\mathrm{Bd}(Q)$. Again by hypothesis, there is an orientation-preserving autohomeomorphism $\psi_{3}$ of $S$ which is the identity on either $Q$ or $S-\operatorname{Int}(Q)$, and which takes $\psi_{2} \psi_{1}\left(L_{2}\right)$ onto $L_{2}^{\gamma_{2}}$. Evidently, $\psi_{2}$ and $\psi_{3}$ may be so chosen that $\psi_{3} \psi_{2} \psi_{1}\left(K_{\alpha}\right)=\varepsilon_{\alpha} K_{p(\alpha)},(\alpha=1, \ldots, \mu)$. Hence, $L$ admits $\gamma$.

Now assume that $L$ is the product of a link $K$ and a prime link $L_{m+1}$, where $K$ is the product of prime factors $L_{1}, \ldots, L_{m}$. It is not difficult to see that $L^{\gamma}$ is the product of a link denoted by $K^{\gamma}$ and the prime link $L_{m+1}^{\gamma_{m}+1}$, where $K^{\gamma}$ is the product of prime factors $L_{1}^{\gamma}, \ldots, L_{m}^{\gamma}$. Then there are 3-cells $Q$ and $Q^{\gamma}$ such that $L$ and $\operatorname{Bd}(Q)$ form a product representation of $L$ with respect to some component $K_{\alpha}$ of $L, L^{\gamma}$ and $\mathrm{Bd}\left(Q^{\gamma}\right)$ form a product representation of $L^{\gamma}$ with respect to the component $\varepsilon_{\alpha} K_{p(\alpha)}$ of $L^{\gamma}$, and such that $K \approx[L \cap \operatorname{Int}(Q)] \cup q, L_{m+1} \approx[L \cap(S-Q)] \cup q$, $K^{\gamma} \approx\left[L^{\gamma} \cap \operatorname{Int}\left(Q^{\gamma}\right)\right] \cup q^{\gamma}$, and $L_{m+1}^{\gamma_{m}+1} \approx\left[L^{\gamma} \cap\left(S-Q^{\gamma}\right)\right] \cup q^{\gamma}$, where $q$ and $q^{\gamma}$ are connecting arcs on $\operatorname{Bd}(Q)$ and $\operatorname{Bd}\left(Q^{r}\right)$, respectively. It is convenient to denote $[L \cap \operatorname{Int}(Q)] \cup q$ by its equivalent $K,[L \cap(S-Q)] \cup q$ by its equivalent $L_{m+1}$, etc.

If now $\psi_{1}$ is an autohomeomorphism of $S$ such that $\psi_{1}(S)=\varepsilon_{0} S$, then by the induction hypothesis $\psi_{1}(K)$ is equivalent to $K^{\nu}$, and there is, therefore, an orientation-preserving autohomeomorphism $\psi_{2}$ of $S$ taking $\psi_{1}(K)$ onto $K^{\gamma}$ and $\psi_{1}(Q)$ onto $Q^{\gamma}$. Since $L_{m+1}$ and $L_{m+1}^{\gamma_{m+1}}$ are of the same type, there is an orientationpreserving autohomeomorphism $\psi_{3}$ of $S$ taking $\psi_{2} \psi_{1}\left(L_{m+1}\right)$ onto $L_{m+1}^{\gamma_{m+1}}$ which acts as the identity on $Q^{\gamma}$. Finally, by the induction hypothesis and the hypothesis of the theorem, $\psi_{2}$ and $\psi_{3}$ can certainly be chosen so that $\psi_{3} \psi_{2} \psi_{1}\left(K_{\alpha}\right)=\varepsilon_{\alpha} K_{p(\alpha)},(\alpha=1$, $\ldots, \mu)$; thus, $L$ admits $\gamma$.

COROLlaRY. If $L=K_{1} \cup K_{2}$ is an unsplittable link, then its prime factors consist of a prime link $L^{*}=K_{1}^{*} \cup K_{2}^{*}$ and a collection $K_{11}, \ldots, K_{1 m}$ and $K_{21}, \ldots, K_{2 n}$ of prime knots, where $K_{1}^{*}$ and $K_{1 \beta}$ are factors of $K_{1}$ and $K_{2}^{*}$ and $K_{2 \beta}$ are factors of $K_{2}$. In order that $L$ be interchangeable it is necessary and sufficient that

(1) $L^{*}$ admit an element $\gamma \in \Gamma_{2}$ of the form $\gamma=\left(\varepsilon_{0}, \varepsilon_{1}, \varepsilon_{2},(12)\right)$.

(2) $m=n$, and there are two permutations $q$ and $r$ on $\{1, \ldots, m\}$ together with two autohomeomorphisms $\phi_{\beta}$ and $\theta_{\beta}$ for each $\beta=1, \ldots, m=n$ such that $\phi_{\beta}(S)=\varepsilon_{0} S$, $\theta_{\beta}(S)=\varepsilon_{0} S, \phi_{\beta}\left(K_{1 \beta}\right)=\varepsilon_{1} K_{2 q(\beta)}$, and $\theta_{\beta}\left(K_{2 \beta}\right)=\varepsilon_{2} K_{1 r}(\beta)$.

Remark. The prime link $L^{*}$ of the above corollary will be called the $h u b$ of the link $L$.

2. Covering spaces. Let $M_{g}$ denote the $g$ th cyclic covering space of $S$ branched over the link $L=K_{1} \cup K_{2}$. Let $\tilde{L}=\widetilde{K}_{1} \cup \widetilde{K}_{2} \subset M_{g}$ lie over $L$, where $\widetilde{K}_{i}$ lies over $K_{i}$.

THEOREM 3. The first homology group of $M_{g}-\widetilde{K}_{1}$ is isomorphic to that of $M_{g}-\widetilde{K}_{2}$. 
Proof. The proof is patterned after that of Theorem (6.1) of [5]. Let

$$
\left(x_{0}, \ldots, x_{n_{1}}, y_{0}, \ldots, y_{n_{2}}: r_{0}, \ldots, r_{n_{1}+n_{2}+1}\right)^{\phi}
$$

be an over presentation [12] for the group $G=\pi_{1}(S-L)$, where the abelianizing homomorphism $\psi$ of $G$ given by $x_{i}^{\psi \phi}=t_{1}$ and $y_{j}^{\psi \phi}=t_{2}$ maps $G$ onto the free abelian group $G /[G, G]$ of rank 2 generated by elements denoted by $t_{1}$ and $t_{2}, t_{m}$ being represented by a loop whose linking number with $K_{n}$ is $\delta_{m n}$. We change from the above presentation to

$$
\left(x, a_{1}, \ldots, a_{n_{1}}, y, b_{1}, \ldots, b_{n_{2}}: s_{0}, \ldots, s_{n_{1}+n_{2}+1}\right)^{\phi}
$$

by introducing new generators $a_{j}=x_{j} x_{0}^{-1}, b_{i}=y_{i} y_{0}^{-1}, x=x_{0}, y=y_{0}$, writing

for

$$
s_{k}\left(x, a_{1}, \ldots, a_{n_{1}}, y, b_{1}, \ldots, b_{n_{2}}\right)
$$

$$
r_{k}\left(x_{0}, a_{1} x_{0}, \ldots, a_{n_{1}} x_{0}, y_{0}, b_{1} y_{0}, \ldots, b_{n_{2}} y_{0}\right)
$$

and $\phi$ again for the canonical homomorphism. Then $x^{\psi \phi}=t_{1}, a_{j}^{\psi \phi}=1, y^{\psi \phi}=t_{2}$, and $b_{i}^{\psi \phi}=1$. By the fundamental formula of the free calculus [3, (2.3)],

$$
\left(t_{1}-1\right)\left(\partial s_{k} / \partial x\right)^{\psi \phi}+\left(t_{2}-1\right)\left(\partial s_{k} / \partial y\right)^{\psi \phi}=0 .
$$

Let $Z$ denote an infinite cyclic group with generator $t$. Let $\tau: G /[G, G] \rightarrow Z$ by $t_{1}^{\tau}=t_{2}^{\tau}=t$ and extend to the group rings. It follows immediately that

$$
\left(\partial s_{k} / \partial x\right)^{\tau \psi \phi}+\left(\partial s_{k} / \partial y\right)^{\tau \psi \phi}=0
$$

Now let $\sigma: t \rightarrow(01 \ldots(g-1))$ be the representation of $Z$ upon $Z_{g}$, (the cyclic subgroup of $S_{g}$ generated by $(01 \ldots(g-1))$, and let $\theta$ be the regular representation of $Z_{g}$ on the group of $g \times g$ matrices. $\rho=\sigma \tau \psi$ is then a transitive representation of $G$ upon $Z_{g}$ corresponding to the unbranched covering space $M_{g}-\tilde{L}$. Now extend $\rho$ and $\theta$ to the group rings.

Then a relation matrix for $H_{1}\left(M_{g}-\widetilde{K}_{2}\right) \oplus A_{g-1}$ ([5] or p. 148 of [1]), where $A_{g-1}$ denotes the free abelian group of rank $g-1$, is the integral matrix

$$
\left\|\left(\frac{\partial\left(s_{0}, s_{1}, \ldots, s_{n_{1}+n_{2}+1}\right)}{\partial\left(x, y, a_{1}, \ldots, a_{n_{1}}, b_{1}, \ldots, b_{n_{2}}\right)}\right)^{\theta \rho \phi}\right\| .
$$

A relation matrix for $H_{1}\left(M_{g}-\widetilde{K}_{1}\right) \oplus A_{g-1}$ is

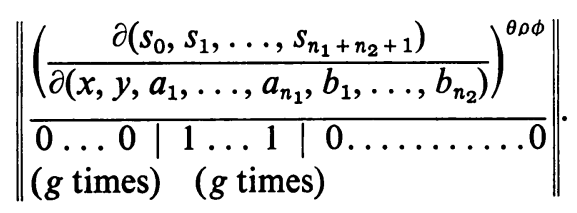


By use of equation $\left(^{*}\right)$, it is clear that each of these matrices is equivalent to

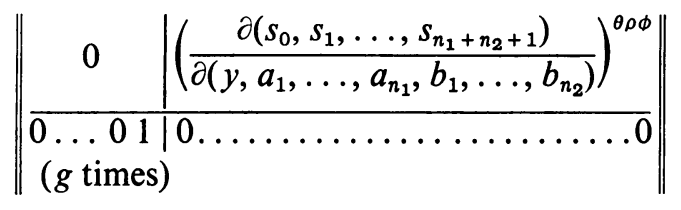

and the theorem follows.

Now consider a link $L=K_{1} \cup \cdots \cup K_{\mu}$, let $M_{g}$ denote the $g$ th cyclic covering space of $S$ branched over $L$, and let $\tilde{L}=\widetilde{K}_{1} \cup \ldots \cup \widetilde{K}_{\mu} \subset M_{g}$ lie over $L$, where as before $\tilde{K}_{i}$ lies over $K_{i},(i=1, \ldots, \mu)$. Finally, let $K_{i}$ and $K_{j}$ be any two components of $L$.

THEOREM 4. The first homology group of $\left(M_{g}-\tilde{L}\right) \cup \tilde{K}_{i}$ is isomorphic to that of $\left(M_{g}-\tilde{L}\right) \cup \tilde{K}_{j}$.

A proof of Theorem 4 may be constructed from that of Theorem 3 with the obvious changes.

Immediately we have an important

COROLlaRY. The group $H_{1}\left(M_{g}-\tilde{L}\right)$ is isomorphic to the direct sum of $H_{1}\left(\left(M_{g}-\tilde{L}\right)\right.$ $\left.\cup \widetilde{K}_{i}\right)$ and the infinite cyclic group for any $i=1, \ldots, \mu$.

If now $L=K_{1} \cup K_{2}$ is interchangeable, then $M_{g}-\tilde{K}_{1}$ is homeomorphic to $M_{g}-\tilde{K}_{2}$, which implies the existence of a one-to-one correspondence between the first homology groups of the $r$-fold unbranched cyclic covering spaces of $M_{g}-\widetilde{K}_{1}$ and those of $M_{g}-\widetilde{K}_{2}$ such that corresponding groups are isomorphic. These $r$-fold unbranched cyclic covering spaces of $M_{g}-\widetilde{K}_{i}$ (see Figure 1) are $r g$-fold branched covering spaces of $S-K_{i}$.

The hubs of the links given in Figures 2(a) and 3(a) are illustrated in Figures 2(b) and 3(b), respectively. Right(left)-handed trefoil knots are denoted by $+(-)$ signs as shown.

If a link of two components is interchangeable, it follows from the corollary to Theorem 2 that the knots $k_{1}=K_{11} \# \cdots \# K_{1 m}$ and $k_{2}=K_{21} \# \cdots \# K_{2 m}$ are of the same knot type. The link of Figure 2(a) is not interchangeable, since for it, $k_{1}$ and $k_{2}$ are of different types (see, for example, [6]). Notice that the components of this link are, however, of the same knot type.

Figure 3(a) shows a link whose hub is interchangeable and for which $k_{1}=K_{11}$ and $k_{2}=K_{21}$ are of the same knot type. This link does not, however, satisfy condition (2) of the corollary to Theorem 2 . Since the trefoil is nonamphichaeral, any autohomeomorphism of $S$ taking $K_{11}$ onto $K_{21}$ must reverse the orientation of $S$. On the other hand, since the hub of the link is also nonamphichaeral [8], any autohomeomorphism of $S$ which interchanges the hub must preserve the orientation of $S$. Hence, the link cannot be interchanged. 
220

W. C. WHITTEN, JR.

[January

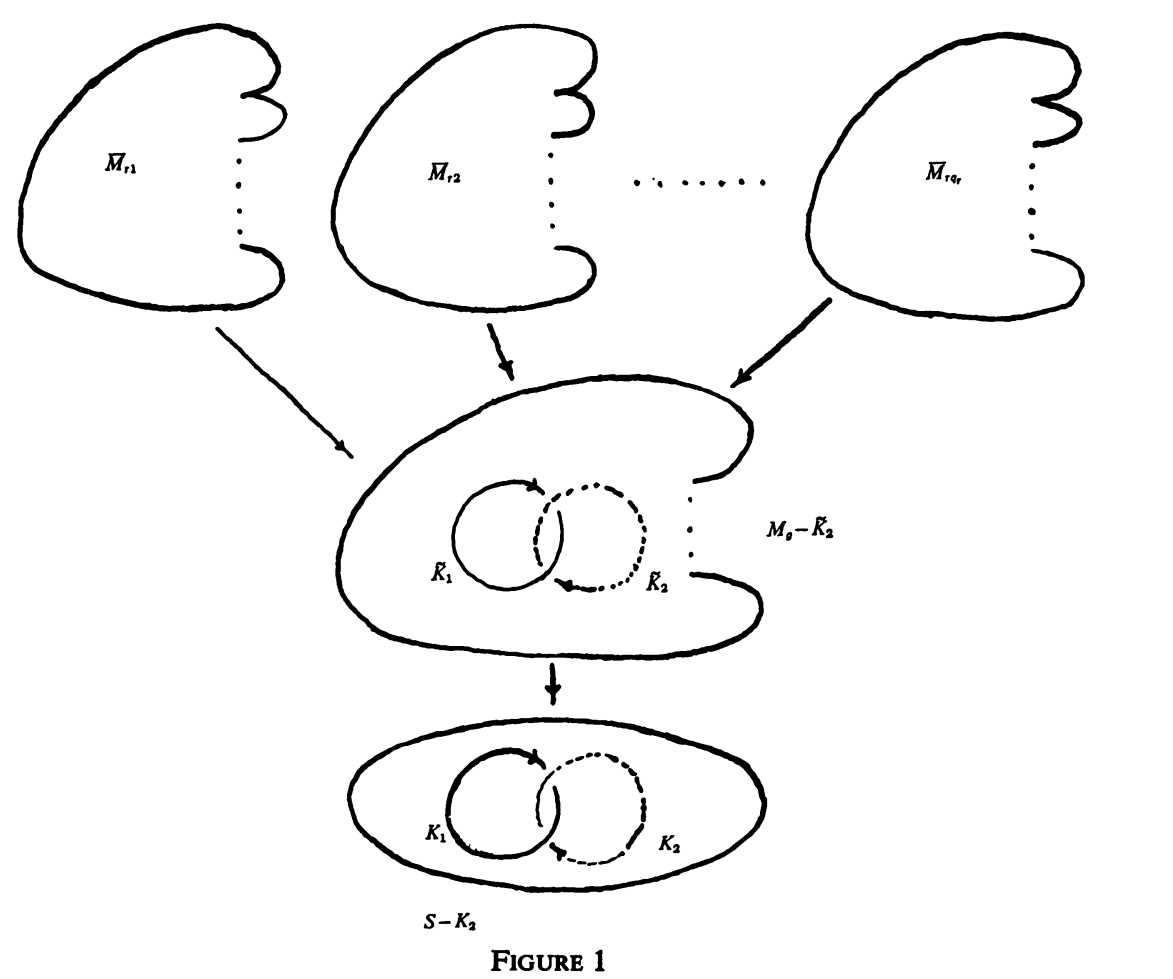

3. Examples.
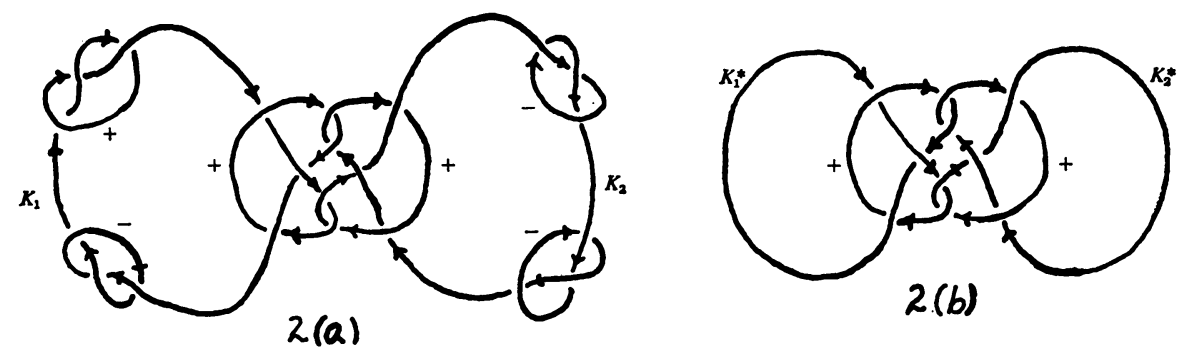

FIGURE 2

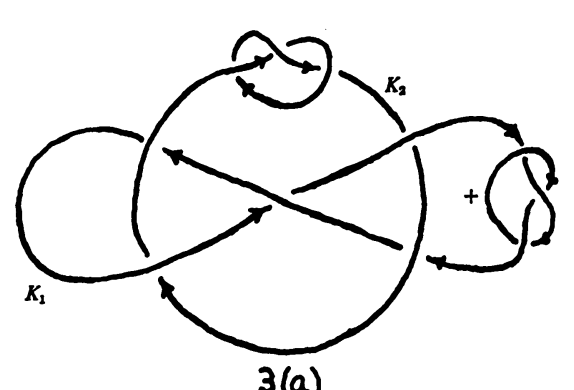

$3(a)$

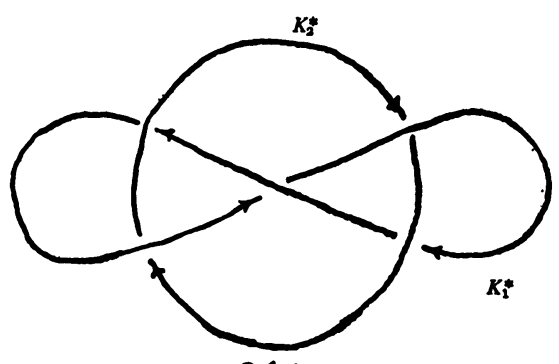

3(b)

FIGURE 3 


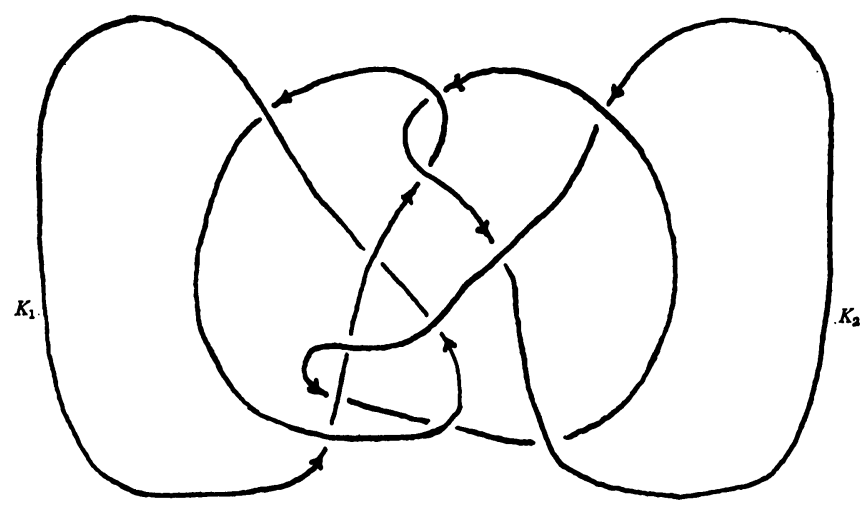

FIGURE 4

Now, consider the link shown in Figure 4. For each of $i=1,2$, there is exactly one two-fold and one three-fold unbranched cyclic covering space $M_{i}^{*}(2)$ and $M_{i}^{*}(3)$, respectively, of $M_{2}-\tilde{K}_{i}$. I calculate that

$$
H_{1}\left(M_{1}^{*}(2)\right) \approx Z \oplus Z_{3} \oplus Z_{51} \approx H_{1}\left(M_{2}^{*}(2)\right) ;
$$

however, $H_{1}\left(M_{1}^{*}(3)\right) \approx Z \oplus Z_{8} \oplus Z_{136}$ and

$$
H_{1}\left(M_{2}^{*}(3)\right) \approx Z \oplus Z_{2} \oplus Z_{2} \oplus Z_{2} \oplus Z_{34},
$$

which shows that our final example is not interchangeable.

Let $\Delta(x, y)$ denote the (normalized) Alexander polynomial [4] of a link $L$ of two components. If $L$ is interchangeable, then $\Delta(x, y)$ must be an associate of at least one of the four $L$-polynomials, $\Delta\left(y^{\varepsilon_{1}}, x^{\varepsilon_{2}}\right)$, where each of $\varepsilon_{1}$ and $\varepsilon_{2}$ is either +1 or -1 . The link of Figure 4 has polynomial

$$
\Delta(x, y)=y\left(x^{4}-2 x^{3}+x^{2}-2 x+1\right)+y^{2}\left(x^{3}-x^{2}+2 x-1\right)+\left(-x^{4}+2 x^{3}-x^{2}+x\right),
$$

which does not have the necessary property.

More generally, if $\Delta\left(x_{1}, \ldots, x_{\mu}\right)$ denotes the (normalized) Alexander polynomial of $L=+K_{1} \cup \cdots \cup+K_{\mu}$, where $x_{i}$ is represented by a loop whose linking number with $+K_{j}$ is $\delta_{i j}$, then in order that $L$ admit $\gamma=\left(\varepsilon_{0}, \varepsilon_{1}, \ldots, \varepsilon_{\mu}, p\right)$, it is necessary that $\Delta\left(x_{1}, \ldots, x_{\mu}\right)$ be an associate of $\Delta\left(x_{p(1)}^{\varepsilon_{1}}, \ldots, x_{p(\mu)}^{\varepsilon_{\mu}}\right)$. Using this condition it is easy to see that for the link $L$ of Figure 4, $\Sigma(L)$ is either trivial or isomorphic to $Z_{2}$.

The nonsymmetric homotopy linking numbers (see p. 633 of [9]) of a link are clearly interchangeability invariants. It is not difficult to see that one of these numbers for the link of Figure 4 is +1 , while experimentation seems to indicate that the other is +3 .

\section{BIBLIOGRAPHY}

1. R. H. Fox, A quick trip through knot theory, Proc. Univ. of Georgia Inst., 1961, PrenticeHall, Englewood Cliffs, N. J., 1962, pp. 120-167.

2. - Some problems in knot theory, ibid., pp. 168-176. 
3. R. H. Fox, Free differential calculus, I. Derivation in the free group ring, Ann. of Math. 75 (1953), 547-560.

4. - Free differential calculus, II. The isomorphism problem, Ann. of Math. 59 (1954), 196-210.

5. - Free differential calculus, III. Subgroups, Ann. of Math. 64 (1956), 407-419.

6. - On the complementary domains of a certain pair of inequivalent knots, Nederl. Akad. Wetensch. Proc. Ser. A 55 (1952), 37-40.

7. Y. Hashizume, On the uniqueness of the decomposition of a link, Osaka J. Math. 10 (1958), 283-300.

8. R. H. Kyle, Branched covering spaces and the quadratic form of a link, Ann. of Math. 59 (1954), 539-548.

9. E. Pannwitz, Eine elementargeometrische Eigenschaft von Verschlingungen und Knoten, Math. Ann. 108 (1933), 629-672.

10. H. Schubert, Die eindeutige Zerlegbarkeit eines Knotens in Primknoten, S.-B. Heidelberger Akad. Wiss. Math. Nat. Kl. 3 (1949), 57-104.

11. - Bestimmung der Primfaktorzerlegung von Verkettungen, Math. Z. 76 (1961), 116-148.

12. G. Torres and R. H. Fox, Dual presentations of the group of a knot, Ann. of Math. 59 (1954), 211-218.

13. H. Trotter, Noninvertible knots exist, Topology 2 (1964), 275-280.

UNIVERSTTY OF SOUTHWESTERN LOUISIANA,

LAFAYETTE, LOUISIANA 